INVESTIGACIONES

\title{
DIVERSIDAD EDUCATIVA EN EDUCANDOS DEL SISTEMA DE PROTECCION SOCIAL "CHILE SOLIDARIO" DE LA COMUNA DE VALPARAISO: RESULTADOS DE UN ESTUDIO EXPLORATORIO*
} Educative diversity in pupil of the system of social protection "Shared in common Chile"
of the commune of Valparaiso: results of an exploratory study

\section{Cristina Julio Maturana}

Escuela de Pedagogía, Pontificia Universidad Católica de Valparaíso. Avenida El Bosque 1290 (Santa Inés), Viña del Mar. Chile. cristina.julio@ucv.cl

\section{Resumen}

El artículo presenta los resultados de un estudio exploratorio sobre la relación entre vulnerabilidad social y educativa en educandos que pertenecen a familias del Programa Puente y cursan segundo ciclo de educación parvularia y primer ciclo de educación básica. La relación se explora desde una perspectiva de educación inclusiva, con el propósito de discutir la "creencia" que plantea que dicha relación es directa, es decir, que por el solo hecho de pertenecer a este segmento socioeconómico tendrían una alta vulnerabilidad educativa, asociada a fracaso escolar. Para adentrarnos en la situación escolar de cada educando y establecer la relación, se construyó un Indice que permitió evidenciar

\begin{abstract}
The article presents the results of an exploratory research on the relationship between social and educational vulnerability in pupils from families that benefit from the Puente Project, and that currently attend the high school early years education, and the first cycle of primary school education. The relationship is analysed from an inclusive education perspective. It aims to examine the belief that this relationship is direct and that the mere fact of pertaining to low socioeconomic segment means a high educational vulnerability, which is associated to failure at school. To this end, an index was built that eventually signalled a great diversity of educational results
\end{abstract}

1 El Sistema de Protección Chile Solidario es una red de protección social que se organiza según la ley N ${ }^{\circ} 19.949$ del 05.06.2004 y que considera acciones y prestaciones para familias y personas en situación de extrema pobreza que consisten en apoyo psicosocial, acceso a diferentes subsidios, entre ellos al de pro retención escolar, para estudiantes de $7^{\circ}$ básico a $4^{\circ}$ medio, de acuerdo a lo establecido en la ley $\mathrm{N}^{\circ} 19.873$, y a un bono de protección familiar mientras estén en una situación de vulnerabilidad social. En palabras del propio Sistema, esta situación dice relación con las condiciones socioeconómicas que ponen en riesgo a las familias, de situarse en una situación de extrema pobreza, al presentarse contingencias a lo largo del ciclo vital tales como cesantía, enfermedades, entre otras.

* Estudio desarrollado durante el año 2007 gracias al financiamiento de la Pontificia Universidad Católica de Valparaíso a través del proyecto DEI-N $188.738 / 2007$. 
una diversidad de resultados educativos en la población estudiada aun cuando pertenecen al mismo grupo socioeconómico, concluyendo que la relación no es directa.

Palabras clave: vulnerabilidad social, vulnerabilidad educativa, fracaso escolar, aprendizaje escolar, diversidad, inclusión, intersectorialidad. in the population analysed even though they belonged to the same socioeconomic segment, leading to the conclusion that the relationship is not direct.

Key words: social vulnerability, educative vulnerability, scholastic failure, scholastic learning, diversity, inclusion, intersectoriality.

\section{INTRODUCCION}

En la última década el sistema educativo chileno ha asumido el reto de lograr una Educación para Todos y lo ha hecho a través de la suscripción a acuerdos internacionales y compromisos derivados de los mismos. En este contexto asume la alianza entre el Movimiento de Educación para Todos (Jomtien 1990, y Dakar 2000) y el Movimiento de Educación Inclusiva (Salamanca 1994, y Salamanca 1999), que surge desde el compromiso que asume la educación especial con el derecho a la educación de todos y todas a través del Movimiento de Educación Inclusiva. Esta alianza reconoce y suscribe que la educación es un derecho humano básico, y no el privilegio de unos pocos (Declaración Universal de los Derechos Humanos, 1948, y Convención sobre los Derechos del Niño, 1989). En definitiva, esta alianza tiene como propósito aunar esfuerzos para hacer realidad el derecho a la educación. Alianza que hace suya y sustenta el presente estudio.

En las tres primeras etapas del último² proceso de reforma (1990-1993; 1994-1999 y 2000-2005) el discurso de estos movimientos internacionales ha ido impregnando progresivamente el sistema escolar y a las personas que en él participan. Sin embargo, la cuarta etapa se inicia el 2006 con heredadas contradicciones y tensiones entre las metas explicitadas en dicho discurso y su forma de organización para materializarlas. La realidad no da cuenta del deber ser y la brecha entre estratos altos y bajos se ha agudizado.

En efecto, si se asume que el sistema escolar es la forma de concreción de la educación como bien público, en tanto institución social que debería garantizar a la sociedad la formación que reciben sus integrantes, como respuesta, apoyo y contribución a los procesos de desarrollo socioeconómico-cultural de la misma (Tezanos 1992), entonces la sociedad chilena está en deuda con los estratos más pobres.

2 En la historia de Chile han existido otros procesos de reforma, se destacan: Expansión del sistema formal de educación la Ley de Instrucción Primaria, la reforma general en el Gobierno de Frei Montalva, la propuesta de la Escuela Nacional Unificada (ENU) del Gobierno de Allende, el proceso de municipalización y la LOCE en el período militar y por último el proceso de reforma de los gobiernos de la Concertación en el que se enmarca el presente estudio. Para profundizar al respecto ver: Iván Núñez, La educación chilena en el período 1945-1990, en Historia de la educación iberoamericana (1945-1992) / coord. por Adriana Puiggrós, Claudio Lozano, Vol. 1, 1995 (Argentina, Bolivia, Brasil, Caribe, Centroamérica, Cuba, Chile, España), ISBN $950-$ 9467-58-8, pp. 231-252. 


\section{ALGUNOS ANTECEDENTES}

A partir de la experiencia territorial en la comuna de Valparaíso y con aprendices de estratos socioeconómicos bajos ${ }^{3}$, se ratifica la evidencia de que ellos y ellas asisten y se concentran en escuelas municipales y, comparativamente, éstas tienen los más bajos resultados educativos, si se consideran los proporcionados por las encuestas de caracterización socioeconómica y las mediciones de aprendizaje escolar, tanto nacionales como internacionales. Desde esta mirada, parece que estos niños y niñas no alcanzarían los requisitos mínimos para cada nivel educativo y en cada sector y subsector de aprendizaje y menos aún lo harían en las etapas organizadas para ello. En definitiva, pareciera que desde una perspectiva cuantitativa estos niños y niñas estarían aprendiendo menos. Sin embargo, si se analizan los resultados de una misma escuela se pueden encontrar evidencias de diversidad en los procesos, niveles de aprendizaje escolar y resultados educativos entre sus aprendices y por lo mismo necesidades educativas diversas, cuestión que pretende develar el estudio a través de un Indice de Vulnerabilidad Educativa de cada Aprendiz (IVEA).

Por otra parte, sobre la base de esta misma experiencia es posible afirmar tres cosas: 1) Que los procesos de intervención de programas estatales, orientados a población infantil de estratos bajos y en situación de vulnerabilidad social, se realizan por sectores, de manera compartimentalizada; 2) Que la intervención se realiza en una perspectiva de prevención de conductas de riesgo, por lo que pone el acento en las variables psicosociales que pueden vulnerar los derechos del niño, por sobre las variables educativas. 3) Que dichos procesos de intervención se implementan especialmente en escuelas focalizadas por ser éstas las que concentran la población infanto-juvenil de estratos bajos y, por ende, de mayor vulnerabilidad social y posibilidades de riesgo en la salud.

La primera y segunda afirmación se evidencian en que el Sistema de Protección Social Chile Solidario, coordinado desde el Ministerio de Planificación Nacional (MIDEPLAN), atiende e interviene, por una parte, a las familias del primer quintil, a través del Programa Puente y recientemente a los niños y niñas entre 0 y 8 años de edad, a través del Programa Crece Contigo ${ }^{4}$. Su énfasis está en la protección social del núcleo familiar, articulando

3 Ver Informe de Asesoría Técnico Pedagógica a Proyectos de Reescolarización, PUCV (Julio 2005). Ver trabajo de tesis titulado: La Función del profesor de Educación Diferencial en la evaluación de niños, niñas y adolescentes en vulnerabilidad social en instancias de educación no formal: Un estudio cualitativo (Álvarez y otras 2006) guiada por la profesora Julio. Ver informes de práctica profesional a estudiantes que han optado por trabajar en contextos de pobreza (2005 y 2006). Ver CD del $4^{\circ}$ Encuentro Internacional de Educación Especial desarrollado en la ciudad de Talca, la ponencia titulada: Niños, niñas y adolescentes fuera del sistema escolar: diversidad injusta y un compromiso pendiente en la política de educación especial (Julio 2005). Ver $\mathrm{CD}$ del $5^{\circ}$ Encuentro Internacional de Educación Especial desarrollado en la ciudad de Talca, la ponencia titulada: Índice de Vulnerabilidad Educativa: un aporte de la educación especial a la inclusión escolar y social de niños y niñas del Sistema de Protección Social Chile Solidario de la comuna de Valparaíso (Julio 2007).

4 Chile Crece Contigo es un Sistema de Protección Integral a la Primera Infancia que tiene como misión acompañar, proteger y apoyar integralmente, tanto a todos los niños, niñas y sus familias, a través de acciones y servicios de carácter universal, como focalizando "a cada quien según sus necesidades". Su propósito es atender las necesidades y apoyar el desarrollo en cada etapa de la primera infancia (desde la gestación hasta los 4 años), promoviendo las condiciones básicas necesarias, en el entendido de que el desarrollo infantil es multidimensional y, por tanto, simultáneamente influyen aspectos biológicos, físicos, psíquicos y sociales del niño/a y su entorno. Forma parte del Sistema de Protección Social comprometido por el Gobierno de aquí al 2010 y está en línea con los compromisos asumidos por el Estado de Chile al ratificar, en 1990, la Convención Internacional sobre los Derechos del Niño. 
los diferentes sectores sociales desde una perspectiva fundamentalmente psicosocial de satisfacción de necesidades básicas y de promoción social. Lo mismo ocurre con los programas del Ministerio del Interior, orientados a prevenir conductas de riesgo como delincuencia y consumo, los del Ministerio de Salud orientados a prevenir conductas de riesgo en la salud de la población infanto-juvenil y el desarrollo de una vida saludable. Los del Ministerio de Justicia, que a través del Servicio Nacional de Menores (SENAME) se hace cargo de velar por el cumplimiento de los Derechos del Niño.

La tercera afirmación dice relación con que el sistema escolar en general y la escuela en particular son el espacio privilegiado de intervención de la política social, pues es allí donde la población infantil y juvenil se encuentra cautiva y permite un fácil acceso a ella, facilitando el desarrollo de programas como los ya señalados y otros. Vale decir, la escuela se transforma en un instrumento de la política social, relegando la política educativa y con ella las posibilidades de desarrollo y aprendizaje de los educandos. En efecto, la política de cada sector "baja" directamente a la escuela, por lo que es posible encontrar varios proyectos aplicándose simultáneamente y en muchas ocasiones de manera paralela y con débil articulación con la política educativa. Tal situación se agudiza aún más si se considera que el propio sector educación, a través de la JUNAEB, desarrolla e implementa en las escuelas programas psicosociales para compensar las necesidades básicas insatisfechas en los estudiantes, que pudieran afectar su desarrollo y rendimiento escolar, como alimentación, salud, habilidades para la vida, entrega de útiles escolares, entre otros. También este sector se hace cargo de programas asociados a objetivos transversales (consumos, sexualidad, convivencia, deporte y recreación, etc.).

Al mismo tiempo, el mismo Ministerio de Educación desarrolla en estas mismas escuelas, focalizadas, una serie de programas ${ }^{5}$ orientados a asegurar la implementación del currículum prescrito para cada nivel educativo, de preferencia en el primer ciclo, tanto de enseñanza básica como en enseñanza media. Programas que se han centrado por una parte en la gestión pedagógica y por otra en el desempeño docente, ambos para mejorar la calidad de la enseñanza que permita asegurar los resultados educativos esperados por currículo.

Esta intervención masiva concentrada en las escuelas focalizadas ha generado en ellas una tensión entre lo pedagógico y lo psicosocial pues se ha dicotomizado e hipertrofiado en ellas la oferta programática, concentrándose preferentemente en lo psicosocial por sobre lo pedagógico. Se ha asumido que mientras las necesidades básicas no estén satisfechas no se puede aprender, o bien se dificulta el aprendizaje del currículo escolar. Con ello se deja relegada a un segundo lugar la labor pedagógica de la escuela y profesores. Esto ha traído consigo, entre otras, externalización de las responsabilidades profesionales de los docentes y de la escuela y, al mismo tiempo, bajas expectativas ${ }^{6}$ de éstos respecto de sus estudiantes, pues asumen que por el solo hecho de pertenecer a estratos socioeconómicos bajos los niños y niñas no aprenderán. Lo anterior conlleva en los estudiantes y sus núcleos familiares una desesperanza aprendida, pues independiente de lo que hagan

5 Programa Enlaces, Bibliotecas de Aula, Monitores Talleres de Aprendizaje (TAP), Campaña Lectura Escritura y Matemática (LEM), Red de maestros, Gestión "Cascada", etc.

6 Sobre las expectativas docentes y su influencia en el desarrollo intelectual y rendimiento escolar de sus estudiantes, ver teoría sobre el "efecto Pigmalión" en la escuela, con la que Robert Rosenthal (1968) explica que el maestro actúa convirtiendo sus percepciones sobre cada alumno en una didáctica individualizada que le lleva, constructiva o destructivamente, a confirmar esas percepciones. 
en la escuela, éstos fracasarán en ellas, con lo que se cierra el círculo vicioso y perverso en el proceso escolar de estratos bajos.

Sobre la base de lo expuesto, el estudio asume que la política pública de educación no ha sido capaz de integrar los aspectos psicosociales con los procesos pedagógicos y revertir la situación descrita, lo que habría agudizado la inequidad en la que nos encontramos sumidos.

En definitiva, el debate de las políticas públicas del sector educación, sobre las causas que explican esta situación de inequidad, puso énfasis, durante la década de los noventa, en las condiciones de las escuelas y en especial en aquellas focalizadas, por ello se otorgaron los soportes necesarios para desarrollar un óptimo proceso educativo.

En gran medida factores como la infraestructura, el financiamiento de la subvención, la gestión escolar y el rol de los sostenedores, entre otros, han sido abordados y a pesar de que existen cuestiones estructurales y de gestión aún pendientes, tal como se observa en el Informe del Consejo Asesor Presidencial para la Calidad de la Educación (2006) ${ }^{7}$ y el debate surgido de éste, sobre la Ley General de Educación (LEGE), la mayor parte de éstos están resueltos. Ellos pueden ser asumidos como una constante, para poder examinar otras variables que pueden estar incidiendo en los desempeños escolares, de los niños y niñas de estratos bajos y en situación de vulnerabilidad social.

Es precisamente la búsqueda de otros factores, como el de diversidad, en los que se centra el presente estudio, buscando develar, a través de un índice, una diversidad educativa en los aprendices que pertenecen a familias del programa Puente.

\section{EL OBJETO DE ESTUDIO A EXPLORAR: VULNERABILIDAD EDUCATIVA DEL APRENDIZ (VEA)}

La Vulnerabilidad Educativa de cada Aprendiz (VEA) es el objeto que se pretende explorar a través de un índice. El estudio asume una concepción que restringe la vulnerabilidad educativa del educando a la fragilidad que pueden tener los niños y niñas, como legítimos aprendices, de fracasar en el sistema escolar para lograr las metas que éste les impone, a través de formas prescritas por ley y por el currículo nacional explícito ${ }^{8}$.

7 Organismo consultivo integrado por un amplio marco de personalidades ligadas al campo de la educación. Se encargó a dicho consejo una agenda de deliberaciones y estudios que expresaba la decisión del Ejecutivo de formular un conjunto de iniciativas y propuestas relativas a la institucionalidad, las regulaciones y la gestión del sistema escolar. Las recomendaciones fueron entregadas en diciembre de 2006. Para estudiar dichas sugerencias y adoptar decisiones al respecto, el Gobierno conformó, además, un consejo de ministros que ha estudiado y coordinado un conjunto de iniciativas legales y administrativas, que tienen como referente las sugerencias del consejo asesor mencionado. Teniendo como norte el mejoramiento de la calidad de la educación para todos, se envió a la Cámara de Diputados un proyecto de reforma constitucional dirigida a equilibrar el derecho a la educación con la libertad de enseñanza y a asegurar el derecho a una educación de calidad. Asimismo, se envió al Congreso un proyecto que deroga la antigua LOCE y que propone una nueva Ley General de Educación a la que se le agregaron indicaciones fruto del acuerdo entre el Gobierno, la Concertación (coalición de Gobierno) y la Alianza (coalición de oposición al Gobierno) para mejorar esta iniciativa y la iniciativa que crea la Superintendencia de Educación, de modo de contar con instrumentos de fiscalización.

8 Prescrito en la, aún vigente, Ley Orgánica Constitucional de Enseñanza (LOCE); en el currículum escolar que de ella deriva y en el que se establecen los Objetivos Fundamentales Transversales (OFT), Objetivos Fundamentales y Contenidos Mínimos Obligatorios (OF-CMO) para cada nivel educativo y en sus Planes y 
Como ya se ha indicado, sobre la base de los resultados educativos y de aprendizajes de las evaluaciones nacionales e internacionales, en el sistema escolar y social se ha ido configurando la creencia de que los niños y niñas de alta vulnerabilidad social estarían también, automáticamente, en condiciones de alta vulnerabilidad educativa. Afirmando que la relación entre vulnerabilidad social y educativa sería directa debido a que los factores socioeconómicos determinarían su condición de aprendiz y su situación educativa. De ser así, todos los niños y niñas de la comuna de Valparaíso que pertenecen al Programa Puente tendrían la misma situación educativa asociada a fracaso escolar ${ }^{9}$. Hoy no se cuenta con evidencia integrada de ello que permita afirmar o negar esta situación y es el aporte que ha pretendido realizar el estudio.

Sobre la base del enfoque de educación inclusiva indicado, de un paradigma cognitivo social de aprendizaje (Rogoff 1993) y de una perspectiva holística de aprendizaje escolar, sustentado en los planteamientos de Devalle y Vega (1999), O’Brien y Guiney (2003) y Undurraga (2005), el estudio tiene como supuesto que en estos estratos socioeconómicos bajos, al igual que en otros estratos, se puede encontrar diversidad de situaciones escolares que pueden configurar una curva de distribución normal y que, por lo mismo, la relación no sería directa, vale decir, el proceso escolar no debería estar determinado por su origen socioeconómico. En efecto, se asume que el aprendizaje escolar es un aprendizaje dinámico que depende del sistema relacional del que el aprendiz forma parte y por ello se explica desde el entrecruzamiento de múltiples factores, tales como: el contexto social, económico, político, histórico, cultural y ambiental en el que están inmersos los aprendices, sus familias y escuelas; las características individuales de cada aprendiz, las de cada profesor y también las del objeto a aprender y, por supuesto, también de las condiciones curriculares, didácticas, organizativo-institucionales y de gestión pedagógica, que conforman el contexto de la institución escolar.

Desde esta mirada inclusiva, cognitiva social y holística quien fracasa no sería sólo el niño o la niña, sino también su familia y comunidad, sus profesores, su escuela, la política educativa, en definitiva, el sistema educativo y la sociedad en su conjunto.

\section{¿POR QUE UN INDICE? JUSTIFICACION TEORICA Y PRACTICA}

La construcción de un Índice de Vulnerabilidad Educativa del Aprendiz (IVEA) ${ }^{10}$ es necesaria porque:

Programas de cada sector y subsector de aprendizaje para cumplir dicho currículo.

9 Se asume el fracaso escolar como un referente para indicar una situación individual o grupal de vulnerabilidad educativa, y lo comprende de manera restringida como el no logro de las metas educativas prescritas a nivel nacional a través de la modalidad organizativa dada por el propio sistema escolar nacional para hacerlo. Desde esta concepción, se fracasa en el logro de metas estandarizadas a través de las formas de organización específicas para hacerlo, también estandarizadas a nivel nacional e internacional. Es así como "el éxito o el fracaso se definen de acuerdo a normas de excelencia sobre las que los alumnos y sus padres (o las familias) poco tienen que decir. Dichas normas configuran una "caja negra" que se les imponen sin darle participación en su contenido" (Devalle y Vega 1999: 90) y sobre las cuales la escuela y el sistema en su conjunto emiten sus juicios respecto de los aprendices y su proceso educativo. Así, el sistema escolar, la escuela y sus integrantes ponen la mirada en la fragilidad educativa del aprendiz, asumiendo que el que fracasa es precisamente éste.

10 El Índice de Vulnerabilidad Educativa del Aprendiz (IVEA) que propone el presente estudio se centra en el aprendiz en el contexto del proceso pedagógico y por lo mismo se diferencia del Índice de Vulnerabilidad 
1. Puede ser un medio que facilite el romper la barrera de la homogeneización que invisibiliza las individualidades, o dicho en términos positivos, podría ser un medio que favorece la visibilización de la diversidad de educandos en el aula, en la escuela, en el sistema educativo y social.

2. Puede ser un instrumento que permita complementar información acerca de las variables biopsicosociales con aquellas pedagógicas propias de los procesos educativos formales.

3. El estado del arte en la búsqueda de factores que puedan explicar el fenómeno educativo de niños y niñas de estratos socioeconómicos bajos, y la inequidad educativa aún presente en Chile ha dado cuenta de factores como:

- La cultura escolar, la gestión escolar, las prácticas pedagógicas y en todos ellos se puso el acento en la incidencia de estas variables en los procesos de enseñanza (CIDE y PIIE, años 80);

- La práctica educativa de profesores, directivos y el esfuerzo de la comunidad escolar como el factor de mayor incidencia en los y las estudiantes que alcanzan mayores aprendizajes en escuelas focalizadas, denominadas efectivas (Bellei y otros 2003). De este estudio publicado por UNICEF derivan guías de apoyo para profesores y profesoras "Buenas Prácticas para una Pedagogía Efectiva" (2005) es posible afirmar que se reconoce como uno de los factores influyentes en los aprendizajes aquellos derivados del manejo de la diversidad en el aula, es decir aquellos de una pedagogía de la diversidad. Sin embargo, se evidencia en ellos una concepción restringida de diversidad y como un principio orientador, sin dar cuenta de elementos pedagógicos y didácticos para su implementación en el aula, como tampoco el por qué existe y cómo se genera dicha diversidad y menos aún las características peculiares de los procesos educativos de los niños y niñas en situación de vulnerabilidad socioeconómica.

- La implementación curricular en el aula. Una serie de estudios realizados por la Unidad de Seguimiento de la Unidad de Currículum y Evaluación UCE del Ministerio de Educación del año 2000 y 2002 nominado Escuelas Testigo, ha realizado un seguimiento a dicha implementación. Considerando los contenidos curriculares y algunos elementos pedagógicos curriculares como: el tiempo, ritmo y secuencia de las actividades de enseñanza en relación con los resultados del SIMCE obtenidos por cada escuela. Esta opción deja fuera del estudio la consideración de los aprendices y sus características educativas en contextos de pobreza.

- La carencia de formación temprana en los escolares ha sido una de las variables recientemente incluida, tanto en el debate de políticas públicas como en el acadé-

Escolar o IVE establecimiento(IVE) y del Índice de Bienestar Infantil (IBI), ambos instrumentos creados y utilizados por la Junta Nacional de Auxilio Escolar y Becas (JUNAEB), servicio del Ministerio de Educación en Chile. El primero es para una medición anual que realiza este organismo estatal, en el mes de abril, mediante la aplicación de una encuesta de carácter censal a los primeros básicos y primeros medios de los establecimientos educacionales, municipales y particulares subvencionados, que postulan al Programa de Alimentación Educativa (PAE) y el segundo, recientemente elaborado que con una visión más amplia biopsicosocial incorpora factores de riesgo y factores protectores desde una perspectiva de los niños, niñas y jóvenes que se encuentran en establecimientos educativos subvencionados. 
mico $^{11}$. Ésta podría explicar los magros desempeños posteriores en la educación básica y media. La falta de acceso a la educación parvularia o su ingreso tardío a la misma sería un factor que no permitiría capitalizar el efecto acumulativo que tendría la intervención oportuna en períodos críticos y sensitivos de desarrollo o, mejor dicho, las "ventanas de oportunidades" para aprendizajes ${ }^{12}$. La cobertura de educación parvularia aparece hoy como la solución central a implementar para hacerse cargo del problema de la educación en general, enfatizando que la calidad de la educación infantil es un medio para disminuir las exclusiones y desigualdades sociales.

- Los pocos docentes capacitados en diversidad sería otro factor que ha aparecido en el discurso público. En efecto, en el discurso presidencial del 21 de mayo (2006) es S.E. la Presidenta Bachelet quien indica que "Sin docentes realmente capacitados para formar niños de orígenes diversos no es posible avanzar en calidad". Al respecto, también se plantea Juan Eduardo García-Huidobro ${ }^{13}$ y algunos programas de fortalecimiento pedagógico ${ }^{14}$.

4. A la fecha aún no se ha dado cuenta del factor diversidad y de las barreras al aprendizaje que se generan al invisibilizar aquellas diferencias derivadas de orígenes socioeconómicos. El estudio asume que mayor cobertura en la educación formal no garantiza que los niños y niñas lleguen a ser incluidos (valorados en sus diferencias) en la educación parvularia, como tampoco en la educación básica. Tampoco aquellos que se incorporen en forma más temprana tienen garantizado un desarrollo cognitivo tal que les permita conocimientos y aprendizajes que sean valorados en el sistema escolar o que éste tenga una sensibilidad en el que les permita identificar sus necesidades educativas para enfrentar con éxito el proceso escolar o superar las dificultades escolares que pudiesen enfrentar como aprendices de estratos socioeconómicos bajos.

Desde una perspectiva de educación inclusiva y sobre la base de los resultados educativos evidenciados en Chile en los últimos años y del estado del arte de la educación referida a niños y niñas de estratos bajos, parecería que la peculiaridad de los procesos educativos de estos niños y niñas no estaría siendo valorada por el sistema escolar, tampoco considerada por los profesores en sus procesos de enseñanza y tampoco en la formación inicial y permanente de profesores de la primera infancia. La explicación de ello no estaría lo suficientemente acabada, quizá sería porque no las conocen o bien, si lo hacen, su valoración sería negativa. No aprenderían como ya se indicó, precisamente

11 Ver discurso de S.E. la Presidenta de la República el 21 de mayo de 2006. Seminario "Educación de Calidad para un Buen Comienzo", Mineduc 2006. El Informe de la comisión asesora Presidencial de Infancia. Las directrices y sustento del Programa Crece Contigo orientado a la primera infancia ( 0 a 8 años de edad) del Sistema de Protección Social Chile Solidario.

12 Peralta, M.V. (2002). Una Pedagogía de las oportunidades. Nuevas Ventanas para los Párvulos Latinoamericanos del siglo XXI, Santiago de Chile: Editorial Andrés Bello, pp. 68-76.

13 García -Huidobro, J.E. (2006). Formación Inicial de Educadoras(es) de Párvulos en Chile, presentado en Seminario "Educación de Calidad para un Buen Comienzo". Santiago: Mineduc y publicado en Foco $\mathrm{N}^{\mathrm{o}} 80$.

14 Programas de Fortalecimiento Pedagógico de Educadoras de Párvulos Mineduc-CPEIP con Apoyo de Universidades. Perfeccionamientos internos JUNJI-INTEGRA, entre otros. Construcción de saberes pedagógicos desde la Implementación de la Reforma en Educación Parvularia, Mineduc-CPEIP (2006). 
por pertenecer a estos estratos bajos. Su situación socioeconómica y sociocultural, incidiría, según algunos, con un énfasis más clínico y patológico, en problemas de desarrollo psicológico y de aprendizaje en los propios estudiantes y, según otros, más contextuales y culturales, pero igualmente negativos en su valoración de los procesos educativos de estos aprendices, incidiría en "las bajas expectativas que los docentes tienen respecto a las posibilidades de aprendizaje y de educabilidad de sus jóvenes estudiantes por su condición de pobreza" (Dávila y otros 2005:14).

En definitiva, se justifica ocuparse, desde la educación especial, de la construcción de un Indice de Vulnerabilidad Educativa, porque éste puede ser un instrumento que permita iniciar un proceso de visibilización de las diversas situaciones educativas escolares que pudieran presentar niños y niñas que pertenecen al Sistema de Protección Chile Solidario. Más aún, si se asume el concepto de infancia dado por Maria Da Costa $(2005)^{15}$, no debería haber una situación universal en ellos y ellas. La autora critica la 'universalidad de la infancia' que promueve una forma única y particular de experiencia educativa, dictada por estados y fases sin considerar de igual forma la localidad y focalidad de la experiencia de aprender y conocer, asumiendo y proponiendo un doble paradigma que plantea que la infancia es una construcción social que varía a través del tiempo y del espacio y que los niños y las niñas son agentes activos en la construcción de sus vidas. En suma, cada tipo de infancia estaría regida por el hacer social en donde tiene lugar.

Son pocas las referencias a una visión integrada del niño y la niña de estratos bajos que caractericen su situación escolar desde una perspectiva holística y que al mismo tiempo integre la multiplicidad de factores y sus posibles combinaciones, que pueden estar en juego en el sistema relacional del que forman parte cada uno de ellos y ellas y que configuran sus peculiaridades, su identidad y sus necesidades educativas en el acceso, ingreso y éxito o fracaso en el proceso educativo, en el contexto del sistema escolar chileno actual. Comenzar a visibilizar esta configuración a través de un índice favorece el inicio de una ruptura de barreras a su aprendizaje, contribuye a los saberes profesionales (de formación inicial y continua) de quienes son los responsables de las mediaciones pedagógicas y psicosociales para hacer que éstas sean más pertinentes a las necesidades humanas de los niños y niñas del Sistema de Protección Chile Solidario de la comuna de Valparaíso.

\section{ESTRUCTURA METODOLOGICA}

El IVEA. Por Indice de Vulnerabilidad Educativa de los y las Aprendices (IVEA) se entiende aquel instrumento estadístico que permite medir, resumir y expresar con características homogéneas el fenómeno de la vulnerabilidad educativa con sus múltiples variaciones, en cada aprendiz y grupos de aprendices y lo hace, durante un período de tiempo determinado, en este caso al cierre del primer semestre escolar 2007.

En términos de proceso y en coherencia con el marco referencial, se construye el índice sobre la base de cuatro dimensiones levantadas desde el enfoque holístico de pedagogía y

15 Dra. Maria Da Costa (PhD). School of Early Childhood. Institute of Education The University of London. Seminario Internacional: "Construyendo el futuro de los niños y niñas". Conferencia: Los desafíos de la formación docente para una educación infantil del siglo XXI: post estructuralismo, globalización y sus implicaciones para la educación de la primera infancia. Noviembre de 2005. 
aprendizaje escolar ya expuesto. Vale decir, se estructura considerando: 1. El sujeto que Aprende, 2. El contexto cultural del que proviene el Sujeto que Aprende, 3. El Sujeto que Enseña y 4. El contexto cultural de la escuela ${ }^{16}$ donde se realizan las interacciones. Cada una de estas dimensiones se subdivide en variables que inciden en el proceso de aprendizaje escolar, tal como lo muestra la tabla 1, las que a su vez desagregan factores específicos en cada uno de ellos, los que se transforman en los indicadores nominales y operacionales del instrumento.

\section{Tabla 1}

Dimensiones, variables y sus ponderaciones del instrumento de recolección de información de Educación Parvularia y Educación Básica para la construcción del IVEA

\begin{tabular}{|c|c|c|}
\hline Dimensión & \multicolumn{2}{|c|}{ Variables } \\
\hline El educando & \multicolumn{2}{|c|}{ Factores del sujeto que aprende $(20 \%)$} \\
\hline & $\begin{array}{l}\text { Nivel de logro por Ámbitos de } \\
\text { Aprendizaje en EPA. (35\%) }\end{array}$ & $\begin{array}{l}\text { Promedio de Calificaciones de } \\
\text { Subsectores de Aprendizajes en } \\
\text { EBA }(\mathbf{1 5 \% )}\end{array}$ \\
\hline & $\begin{array}{l}\text { Asistencia al finalizar Primer } \\
\text { Semestre Académico } \mathbf{( 4 0 \% )}\end{array}$ & $\begin{array}{l}\text { Asistencia al finalizar Primer } \\
\text { Semestre Académico } \mathbf{( 2 5 \% )}\end{array}$ \\
\hline & Comportamiento $(\mathbf{2 5 \%})$ & Comportamiento $(\mathbf{1 0 \%})$ \\
\hline & & Repitencia (20\%) \\
\hline & & Sobreedad (20\%) \\
\hline & & $\begin{array}{l}\text { Pertenencia a Grupo Diferencial } \\
(\mathbf{1 0 \%})\end{array}$ \\
\hline El contexto familiar & \multicolumn{2}{|c|}{ Factores culturales del sujeto que aprende (20\%) } \\
\hline & \multicolumn{2}{|c|}{$\begin{array}{l}\text { Organización de la familia (Escala Likert: 1-100\%, 2-80\%, 3-60\%, } \\
\mathbf{4 - 8 0 \%} \text { y 5-100\%) }\end{array}$} \\
\hline & \multicolumn{2}{|l|}{ Ocupación de los padres: Id. } \\
\hline & \multicolumn{2}{|l|}{ Dinámica Familiar: Id. } \\
\hline & \multicolumn{2}{|c|}{ Condiciones mínimas de educación en el grupo familiar: Id. } \\
\hline El profesor & \multicolumn{2}{|c|}{ Factores del sujeto que enseña $\mathbf{( 3 0 \% )}$} \\
\hline & \multicolumn{2}{|l|}{ Edad (5\%) } \\
\hline & \multicolumn{2}{|l|}{ Año de titulación (10\%) } \\
\hline & \multicolumn{2}{|l|}{ Jornada laboral del docente (20\%) } \\
\hline & \multicolumn{2}{|c|}{ Planificación de la sesión de clases del docente (25\%) } \\
\hline & \multicolumn{2}{|l|}{ Experiencia laboral (15\%) } \\
\hline & \multicolumn{2}{|l|}{ Asistencia (25\%) } \\
\hline
\end{tabular}

16 El estudio asume una visión crítica de cultura escolar, por ello la entiende como un conjunto de significaciones compartidas y actitudes, valores y creencias significativas para las personas que configuran la comunidad escolar y que se evidencian en sus modelos de relación, formas de asociación y organización que las distinguen, caracterizan e identifican respecto de otras. 


\begin{tabular}{|l|l|}
\hline \multicolumn{1}{|c|}{ Dimensión } & \multicolumn{1}{c|}{ Variables } \\
\hline El contexto institucional & Factores de la cultura escolar (30\%) \\
\hline & Proyecto Educativo Institucional (PEI) (10\%) \\
\cline { 2 - 3 } & Información del alumnado (20\%) \\
\cline { 2 - 3 } & Supervisión de planificaciones (20\%) \\
\cline { 2 - 2 } & Reuniones Técnicas Docentes (20\%) \\
\cline { 2 - 2 } & Centro de Padres (CCPP) (20\%) \\
\cline { 2 - 2 } & $\begin{array}{l}\text { El colegio cuenta con: Reglamento de Evaluación, Grupo Diferencial } \\
\text { o Aula de Recursos, Proyecto de Integración (PIE), Otros proyectos } \\
(\mathbf{1 0 \%})\end{array}$ \\
\hline
\end{tabular}

Los indicadores que forman parte del instrumento fueron levantados sobre la base de una revisión bibliográfica y análisis de las conceptualizaciones educativas y escolares asociadas. En un primer momento éstos fueron depurados sobre la base de las fuentes de información disponibles en el sistema educativo para acceder a la información requerida, en segundo lugar, sobre la base de la opinión de expertos que evaluaron el instrumento, uno en estadística y el segundo, en los niveles educativos correspondientes y finalmente validados en dos establecimientos con educandos del programa.

Para recoger la información se trabajó con dos instrumentos con formato de celdas, uno para EPA y otro para EBA. Se trabaja preferentemente con fuentes secundarias, vale decir, registros proporcionados por los establecimientos del niño o la niña, libros de clases y fichas escolares. Solo se utilizan fuentes primarias (profesores, jefes técnicos o encargados del Programa Puente) en los casos que fue necesario verificar, complementar o completar información relevante. Los antecedentes educativos y culturales de la familia de la cual proviene el niño y la niña, se recogieron de la ficha técnica familiar con la que cuenta el Programa Puente a nivel comunal, proporcionado por la Ilustre Municipalidad de Valparaíso y sobre la base de indicadores de gestión del programa: Cumplido; Cumplido por el programa; A trabajar mínimo pendiente; No corresponde y Sin abordar.

Determinación de la población: Los niños y las niñas. El instrumento se aplica a una población de 370 niños y niñas, 352 de EBA y 18 de EPA. Cabe señalar que para determinar el número de aprendices se optó por un muestreo no probabilístico, debido a que no se contaba con información anterior. Se llega a este número luego de un filtro y organización de la información de la población. Proceso que se da en seis etapas, a saber:

- La primera, depurar la Base de Datos (a octubre 2006) de las personas del Programa Puente de la comuna de Valparaíso, proporcionada por la coordinación provincial del mismo. El primer filtro permite acceder a la población infantil entre 0 y 13 años de edad, lo que redujo la población de 14 mil personas a 4 mil aproximadamente. La opción de los 13 años fue sobre la base del criterio de cobertura, considerando la edad cronológica correspondiente a todo el ciclo de educación básica. Aunque el propósito era trabajar sólo con segundo ciclo de Educación Parvularia, se mantuvieron los 0 años como una primera aproximación a la etapa infantil y el primer ciclo de 
EBA (de $1^{\circ}$ a $4^{\circ}$ ), se amplió la edad a 13 años considerando las posibilidades de sobreedad de algunos estudiantes.

- La segunda, ubicar en el sistema escolar a los niños y niñas identificados, ¿en qué escuela estaban? Se cruza, por Rut, la información obtenida inicialmente, con una base de datos proporcionada, esta vez, por la JUNAEB y el Programa de Alimentación Complementaria (PAC), referida a niños y niñas de EBA a diciembre de 2006, acotando la información a este grupo.

- La tercera, ubicar en el sistema escolar ${ }^{17}$ a los párvulos que cursan segundo ciclo de EPA. Por lo mismo, en este momento, se trabaja con la ficha (en papel) de cada uno de ellos proporcionada por la Coordinación Regional de EPA buscando uno a uno, a través del Rut.

- La cuarta, organizar una Base de datos final, que permite determinar una población de 650 niños y niñas que pertenecen a 29 escuelas de la comuna de Valparaíso y que cursarían los ciclos antes indicados.

- En quinto lugar, se aplica el criterio de establecimientos que concentren 10 o más niños y niñas del Programa Puente.

- Finalmente y en sexto lugar, se ajusta nuevamente la base de datos original (650) por encontrar discrepancia de información al momento de llegar a los establecimientos, debido fundamentalmente a la diferencia de tiempo, información 2006 y aplicación, octubre de 2007. Por lo mismo, la nueva base de datos deja fuera 280 estudiantes, 111 por pertenecer a establecimientos que no participaron del estudio y 169 porque o no se encontraban en el establecimiento o habían sido promovidos. Los 370 aprendices pertenecen a 23 establecimientos de la comuna, 5 de dependencia Particular subvencionada y 18 de dependencia municipal.

Ponderación de variables. Como ya se indicó, en el índice se consideran cuatro dimensiones de un proceso pedagógico holístico: el aprendiz, el profesor, en contexto social y cultural y el contexto institucional. Cada una de ellas tiene una ponderación dada por su influencia en el proceso educativo desde una perspectiva del aprendiz y de sus peculiaridades. Desde una perspectiva interaccional, todas las variables tendrían la misma incidencia en el proceso educativo, en el bien entendido que lo relevante no es el peso específico de cada una de ellas sino su visibilización, para permitir, a partir de ella, su interacción y articulación. Sin embargo, por ser la entidad educativa y los y las docentes los responsables profesionales de contextualizar y adecuar el currículo escolar a las características de los educandos, se elevan las ponderaciones de las dimensiones profesor y escuela, tal como se observa en la tabla 1 ya presentada.

Metodología para el cálculo del IVEA de EPA y EBA. La metodología se presenta por dimensión, organizada en una tabla de doble entrada en la que se presenta paralelamente cada variable y la modalidad de cálculo por nivel educativo y de manera paralela. A continuación se presentan cuatro tablas, en cada una de ellas se da cuenta de una dimensión.

17 Se consideran párvulos que cursan segundo ciclo de EPA en escuelas básicas. No se consideran jardines infantiles. 


\section{Tabla 2}

Metodología para el cálculo de la Primera Dimensión: El Educando Los Factores del sujeto que aprende

\begin{tabular}{|c|c|c|}
\hline $\begin{array}{l}\text { Nivel educativo/ } \\
\text { variables }\end{array}$ & $\begin{array}{c}\text { Educación Parvularia } \\
\text { EPA }\end{array}$ & $\begin{array}{l}\text { Educación Básica } \\
\text { EBA- NB1 }\end{array}$ \\
\hline $\begin{array}{l}\text { Promedio de } \\
\text { Calificaciones de } \\
\text { Subsectores de } \\
\text { Aprendizajes en } \\
\text { NB1 y ámbitos en } \\
\text { EPA }\end{array}$ & $\begin{array}{l}\text { Nivel de logro por Ámbitos de } \\
\text { Aprendizaje en Educación Par- } \\
\text { vularia: } \\
\text { Para cada ámbito se calculó el } \\
\text { porcentaje de rendimiento, siendo } \\
\text { un } 100 \% \text { si lo logra y } 50 \% \text { si lo } \\
\text { logra medianamente y un } 0 \% \text { si } \\
\text { no lo logra. Luego, se calcula el } \\
\text { promedio de los tres ámbitos. }\end{array}$ & $\begin{array}{l}\text { Se calculó el porcentaje que } \\
\text { cumplían los alumnos según sus } \\
\text { calificaciones de la siguiente } \\
\text { manera: } \\
1-((7,0-\text { Promedio }) / 6,0) * 100 \\
\text { Siendo } 7,0 \text { la nota máxima, } 6,0 \text { el } \\
\text { rango de los datos, para las notas } \\
\text { sobre } 6,0 \text { se les asignó el } 100 \text { de } \\
\text { rendimiento. Luego se calculó } \\
\text { el promedio de los subsectores } \\
\text { para generar el indicador de ren- } \\
\text { dimiento. }\end{array}$ \\
\hline $\begin{array}{l}\text { Asistencia al } \\
\text { finalizar Primer } \\
\text { Semestre } \\
\text { Académico }\end{array}$ & $\begin{array}{l}\text { Para esta variable se calculó el por- } \\
\text { centaje de cumplimiento de cada } \\
\text { aprendiz considerando un mínimo } \\
\text { de } 80 \% \text { y un máximo de } 100 \% \text { de } \\
\text { la siguiente manera: } \\
(100 \text { - asistencia) / } 20 \\
\text { Donde } 20 \text { es el rango considerado } \\
\text { entre el mínimo y máximo. A los } \\
\text { aprendices con menos de } 80 \% \text { de } \\
\text { asistencia se les asigna un } 0 \% \text {. }\end{array}$ & Id. \\
\hline Repitencia & No se consideró en este nivel & $\begin{array}{l}\text { Si el alumno no ha repetido se } \\
\text { le asigna un } 100 \% \text {; en caso que } \\
\text { presente repitencia un } 0 \% \text {. }\end{array}$ \\
\hline Comportamiento & $\begin{array}{l}\text { Se asignó un rango de } 0 \text { a } 5 \text { para } \\
\text { los registros positivos y negati- } \\
\text { vos, calculando el porcentaje que } \\
\text { cumplían los educandos en forma } \\
\text { positiva y negativa, para luego } \\
\text { calcular el promedio de estos dos } \\
\text { indicadores, el que finalmente es } \\
\text { utilizado en el índice. }\end{array}$ & Id. \\
\hline Sobreedad & No se consideró en este nivel & $\begin{array}{l}\text { Si no presenta sobreedad se le } \\
\text { asigna un } 100 \% \text {, si el alumno } \\
\text { presenta un año de sobreedad, un } \\
50 \% \text { y, finalmente, si presenta dos } \\
\text { años de sobreedad, un } 25 \% \text {. }\end{array}$ \\
\hline $\begin{array}{l}\text { Pertenencia a } \\
\text { Grupo Diferencial }\end{array}$ & No se consideró en este nivel & $\begin{array}{l}\text { Si el alumno ha pertenecido a un } \\
\text { grupo diferencial durante el presen- } \\
\text { te año o antes, se asigna un } 0 \% \text {, de } \\
\text { lo contrario un } 100 \% \text {. }\end{array}$ \\
\hline
\end{tabular}


Tabla 2

Metodología para el cálculo de la Segunda Dimensión: El contexto Familiar. Factores culturales del sujeto que aprende

\begin{tabular}{|c|c|c|}
\hline Respuesta & Indicador Programa Puente & Porcentaje \\
\hline 1 & Cumplido & $100 \%$ \\
\hline 2 & Cumplido por programa & $80 \%$ \\
\hline 3 & A trabajar mínimo pendiente & $60 \%$ \\
\hline 4 & No corresponde & $40 \%$ \\
\hline 5 & Sin abordar & $20 \%$ \\
\hline
\end{tabular}

Tal como se observa en la tabla 2, esta dimensión consideró en el instrumento aplicado a ambos niveles, las variables indicadas en tabla 1: Organización de la familia, Ocupación de los padres, Dinámica Familiar y Condiciones mínimas de educación en el grupo familiar. Información recopilada de la Ficha Familiar del Programa Puente y organizadas en preguntas en escala Likert. Por ello, metodológicamente se trabajó exactamente igual con la información obtenida en EPA y EBA, a saber: Se asignaron porcentajes según la escala que se presenta y luego se calcula el promedio por aprendiz.

\section{Tabla 3}

Metodología para el cálculo de la Tercera Dimensión:

El profesor/a Factores del sujeto que enseña

\begin{tabular}{|c|c|c|}
\hline $\begin{array}{l}\text { Nivel educativo/ } \\
\text { variables }\end{array}$ & $\begin{array}{c}\text { Educación Parvularia } \\
\text { EPA }\end{array}$ & $\begin{array}{c}\text { Educación Básica } \\
\text { EBA- NB1 }\end{array}$ \\
\hline Edad & $\begin{array}{l}\text { Considerando un mínimo de } 22 \text { años y un máximo } \\
\text { de } 55 \text { se calculó un porcentaje de la siguiente } \\
\text { manera: }(55-E d a d) / 33 \\
\text { Siendo } 55 \text { el valor máximo y } 33 \text { el rango entre los } \\
\text { límites mínimo y máximo de la edad, si la edad es } \\
\text { mayor que } 55 \text {, el valor del indicador es } 0 \% \text {. }\end{array}$ & Id. \\
\hline Año de titulación & $\begin{array}{l}\text { Al igual que la variable anterior se calculó un } \\
\text { porcentaje considerando como mínimo el año } 2006 \\
\text { y máximo el año 1977, de la siguiente manera: } \\
(100-(2006-\text { Año Titulación) }) \text { / } 29 \\
\text { Donde } 2006 \text { es el mínimo y } 29 \text { es el rango con- } \\
\text { siderado. }\end{array}$ & Id. \\
\hline $\begin{array}{l}\text { Jornada laboral del } \\
\text { docente }\end{array}$ & $\begin{array}{l}\text { Se calculó un porcentaje tomando como mínimo } \\
15 \text { horas y máximo } 30 \text { horas de la siguiente } \\
\text { manera: } \\
((\text { Jornada }-15) / 15) * 100) \\
\text { Donde } 15 \text { es el rango considerado para la va- } \\
\text { riable. }\end{array}$ & Id. \\
\hline
\end{tabular}




\begin{tabular}{|c|c|c|}
\hline $\begin{array}{l}\text { Nivel educativo/ } \\
\text { variables }\end{array}$ & $\begin{array}{c}\text { Educación Parvularia } \\
\text { EPA }\end{array}$ & $\begin{array}{c}\text { Educación Básica } \\
\text { EBA- NB1 }\end{array}$ \\
\hline $\begin{array}{l}\text { Planificación de la } \\
\text { sesión de clases del } \\
\text { docente }\end{array}$ & $\begin{array}{l}\text { Si el docente realiza planificación diaria se asigna } \\
\text { un } 100 \% \text {, si realiza planificación semanal un } 80 \% \text {, } \\
\text { si realiza planificación mensual } 60 \% \text {, si realiza } \\
\text { planificación semestral un } 40 \% \text {, si realiza plani- } \\
\text { ficación anual un } 20 \% \text {. }\end{array}$ & $\begin{array}{l}\text { No se consideró en } \\
\text { este nivel por falta } \\
\text { de consistencia en } \\
\text { la información re- } \\
\text { copilada. }\end{array}$ \\
\hline Experiencia laboral & $\begin{array}{l}\text { Para esta variable se tomo como mínimo dos } \\
\text { años y máximo } 30 \text { años, por lo tanto se calculó } \\
\text { el porcentaje que cumplen los docentes dentro de } \\
\text { este rango de la siguiente manera: } \\
100-((30-\text { Experiencia }) / 28) \\
\text { Siendo } 28 \text { el rango entre el valor mínimo y } \\
\text { máximo. }\end{array}$ & $\begin{array}{l}\text { No se consideró en } \\
\text { este nivel por falta } \\
\text { de consistencia en } \\
\text { la información re- } \\
\text { copilada. }\end{array}$ \\
\hline Asistencia & $\begin{array}{l}\text { Se calculó un promedio entre el porcentaje de } \\
\text { asistencia a clases y el porcentaje de asistencia a } \\
\text { consejo de técnicos. }\end{array}$ & $\begin{array}{l}\text { Se calculó un } \\
\text { promedio entre el } \\
\text { porcentaje de asis- } \\
\text { tencia a clases y el } \\
\text { porcentaje de asis- } \\
\text { tencia a consejo de } \\
\text { técnicos. }\end{array}$ \\
\hline
\end{tabular}

\section{Tabla 4}

Metodología para el cálculo de la Cuarta Dimensión:

Contexto Institucional. Los Factores de Cultura escolar

\begin{tabular}{|c|c|c|}
\hline $\begin{array}{l}\text { Nivel educativo/ } \\
\text { variables }\end{array}$ & $\begin{array}{c}\text { Educación Parvularia } \\
\text { EPA }\end{array}$ & $\begin{array}{c}\text { Educación Básica } \\
\text { EBA- NB1 }\end{array}$ \\
\hline $\begin{array}{l}\text { Proyecto Educativo } \\
\text { Institucional (PEI) }\end{array}$ & $\begin{array}{l}\text { Si el establecimiento cuenta con PEI } \\
\text { se asigna un } 100 \% \text { de lo contrario } \\
\text { un } 0 \% \text {. }\end{array}$ & Id. \\
\hline $\begin{array}{l}\text { Información del } \\
\text { alumnado }\end{array}$ & $\begin{array}{l}\text { Si el establecimiento cuenta con } \\
\text { información organizada del estu- } \\
\text { diante se asigna un } 100 \% \text {, si cuenta } \\
\text { con información no actualizada se } \\
\text { asigna un } 50 \% \text {, si no cuenta con } \\
\text { información un } 0 \% \text {. }\end{array}$ & Id. \\
\hline $\begin{array}{l}\text { Supervisión de } \\
\text { planificaciones por UTP }\end{array}$ & $\begin{array}{l}\text { Si existe supervisión semanal se } \\
\text { asigna un } 100 \% \text {, si es mensual un } \\
75 \% \text {, si es semestral un } 50 \% \text { y fi- } \\
\text { nalmente si es anual un } 25 \% \text {. }\end{array}$ & $\begin{array}{l}\text { Si existe supervisión sema- } \\
\text { nal o mensual se asigna un } \\
100 \% \text {, si existe supervisión } \\
\text { con otra frecuencia se asigna } \\
\text { un } 50 \% \text {, si no existe super- } \\
\text { visión se asigna } 0 \% \text {. }\end{array}$ \\
\hline
\end{tabular}




\begin{tabular}{|c|c|c|}
\hline $\begin{array}{l}\text { Nivel educativo/ } \\
\text { variables }\end{array}$ & $\begin{array}{c}\text { Educación Parvularia } \\
\text { EPA }\end{array}$ & $\begin{array}{c}\text { Educación Básica } \\
\text { EBA- NB1 }\end{array}$ \\
\hline $\begin{array}{l}\text { Reuniones Técnicas } \\
\text { Docentes }\end{array}$ & $\begin{array}{l}\text { Si existen reuniones técnicas docen- } \\
\text { tes semanales se asigna un } 100 \% \text {, } \\
\text { si son mensuales un } 75 \% \text {, si son } \\
\text { semestrales un } 50 \% \text {, finalmente si } \\
\text { son anuales un } 25 \% \text {. }\end{array}$ & $\begin{array}{l}\text { Si se realizan reuniones } \\
\text { técnicas docentes semanales } \\
\text { o mensuales se asigna un } \\
100 \% \text {, si se realizan con otra } \\
\text { frecuencia se asigna un } 50 \% \text {, } \\
\text { si no se realizan un } 0 \% \text {. }\end{array}$ \\
\hline $\begin{array}{l}\text { Centro de Padres } \\
\text { (CCPP) }\end{array}$ & $\begin{array}{l}\text { Si existe CCPP que apoya al colegio, } \\
\text { es apoyado por el colegio y realiza } \\
\text { reuniones semestrales, se asigna un } \\
100 \% \text {, si alguna de estas condicio- } \\
\text { nes no se cumple, se resta un } 25 \% \\
\text { y si no existe centro de padres se } \\
\text { asigna un } 0 \% \text {. }\end{array}$ & Id. \\
\hline $\begin{array}{l}\text { El colegio cuenta } \\
\text { con: Reglamento de } \\
\text { Evaluación, Grupo } \\
\text { Diferencial o Aula de } \\
\text { Recursos, Proyecto de } \\
\text { Integración (PIE), Otros. }\end{array}$ & $\begin{array}{l}\text { Se asignará un porcentaje para cada } \\
\text { una de estas categorías por separado } \\
\text { asignando un } 100 \% \text { si se cumple } \\
\text { y un } 0 \% \text { si no, luego se calcula el } \\
\text { promedio. }\end{array}$ & Id. \\
\hline
\end{tabular}

Interpretación del IVEA. Finalmente, para facilitar el análisis de la situación educativa y aportar a una refocalización del proceso educativo, a partir de ésta, se definieron quintiles. Éstos ordenan a los niños y niñas en cinco grupos iguales en función del valor del Índice, tal como se observa en la tabla 5. Cabe destacar que mientras más alto es el porcentaje del índice obtenido por los aprendices, menor es su vulnerabilidad educativa. A los quintiles se les asignó una denominación que corresponde a las condiciones de educabilidad de cada niño y niña para el desarrollo de sus aprendizajes escolares, lo que los hace más o menos vulnerables al proceso educativo formal. Según esta vulnerabilidad, se establece una prioridad en el proceso educativo y, según sea ésta, se sugiere un tipo de intervención pedagógica.

Relación entre vulnerabilidad educativa y vulnerabilidad social. Las variables de vulnerabilidad social están dadas por el propio Sistema de Protección Social. Vale decir, los niños y niñas del programa Puente son aquellos más vulnerables socialmente, por pertenecer a familias del primer quintil socioeconómico y que han sido focalizadas sobre la base de la Encuesta de Caracterización Socioeconómica Nacional CASEN 2006. Por lo mismo, los niños y niñas tienen el mayor índice de vulnerabilidad social, por pertenecer al estrato socioeconómico más bajo.

El Índice de Vulnerabilidad Escolar o Establecimiento (IVE), utilizado por la JUNAEB, también los tiene focalizados como los niños y niñas prioritarios por la política de auxilio escolar, por ser éstos los más vulnerables socialmente.

Cabe preguntarse entonces:

¿Todos estos niños y niñas, prioritarios por su alta vulnerabilidad social, tienen alta vulnerabilidad educativa? 


\section{Tabla 5}

Niveles de vulnerabilidad de los educandos según el Indice

\begin{tabular}{|c|c|c|c|c|}
\hline Quintil & $\begin{array}{c}\text { Condiciones de } \\
\text { educabilidad }\end{array}$ & Nivel de vulnerabilidad & $\begin{array}{c}\text { Prioridad } \\
\text { educativa }\end{array}$ & $\begin{array}{c}\text { Tipo de proceso educativo y } \\
\text { pedagógico sugerido }\end{array}$ \\
\hline Primero & Deficientes & Totalmente vulnerables & Primera & $\begin{array}{c}\text { Reparativo y permanente } \\
\text { en todas las dimensiones }\end{array}$ \\
\hline Segundo & $\begin{array}{l}\text { Menos que } \\
\text { suficientes }\end{array}$ & Altamente vulnerables & Segunda & $\begin{array}{c}\text { Intensa y permanente } \\
\text { en todas las dimensiones. }\end{array}$ \\
\hline Tercero & Suficientes & Vulnerables & tercera & $\begin{array}{c}\text { Permanente } \\
\text { en todas las dimensiones. }\end{array}$ \\
\hline Cuarto & Más que suficientes & Poco vulnerables & Cuarta & $\begin{array}{c}\text { Permanente } \\
\text { en las dimensiones más } \\
\text { afectadas. }\end{array}$ \\
\hline Quinto & Satisfactorias & No vulnerables & Quinta & Monitoreo semestral \\
\hline
\end{tabular}

La respuesta a esta interrogante se entregará en la conclusión, luego de que se expongan los resultados obtenidos.

\section{RESULTADOS}

Los resultados del estudio obtenidos a través del Indice de Vulnerabilidad Educativa del Aprendiz (IVEA) serán presentados por nivel educativo. Su presentación se realiza con información general según grupos de educandos. Por respeto al derecho de intimidad y privacidad de los niños y niñas y sus familias, en este artículo no se presentarán los resultados de cada aprendiz.

El IVEA en Segundo Ciclo de Educación Parvularia. En EPA se encuentra como valor mínimo del Indice un 55,17\% mientras que el valor máximo encontrado es de 95,84\%. Para observar el comportamiento del Indice en el nivel y su distribución, se fijan intervalos, calculando la diferencia entre los dos valores indicados (máximo y mínimo), para luego dividirlo por 5, lo que entrega la amplitud del intervalo. Es así como, mientras más alto es el porcentaje obtenido por los aprendices, menor es su vulnerabilidad educativa.

En definitiva, se puede ver en el gráfico que la mayoría de los educandos del nivel se concentra desde el tercer intervalo con porcentajes superiores a 70\%, vale decir, entre el tercer y quinto quintil, por lo tanto se puede afirmar que tendrían en general una baja vulnerabilidad educativa. Sin embargo, también es posible afirmar que existe diversidad de situaciones de vulnerabilidad, las que también se observan en el gráfico y que pueden apreciarse en detalle en la base de datos construida y que, por las razones ya indicadas, no se hacen públicas. 


\begin{tabular}{|c|c|c|c|r|}
\hline Intervalo & Quintil & $\begin{array}{c}\text { Nivel de } \\
\text { Vulnerabilidad }\end{array}$ & Aprendices & \multicolumn{1}{c|}{ Porcentaje } \\
\hline $0,55-0,63$ & primero & $\begin{array}{c}\text { Totalmente } \\
\text { Vulnerables }\end{array}$ & 1 & $5,56 \%$ \\
\hline $0,63-0,71$ & segundo & $\begin{array}{c}\text { Altamente } \\
\text { Vulnerables }\end{array}$ & 0 & $0,00 \%$ \\
\hline $0,71-0,79$ & tercero & Vulnerables & 6 & $33,33 \%$ \\
\hline $0,79-0,87$ & cuarto & Poco Vulnerables & 6 & $33,33 \%$ \\
\hline $0,87-0,95$ & quinto & No Vulnerables & 5 & $27,78 \%$ \\
\hline & & & 18 & $100,00 \%$ \\
\hline
\end{tabular}

Distribución de aprendices de EPA según

Indice de Vulnerabilidad Educativa (IVE)

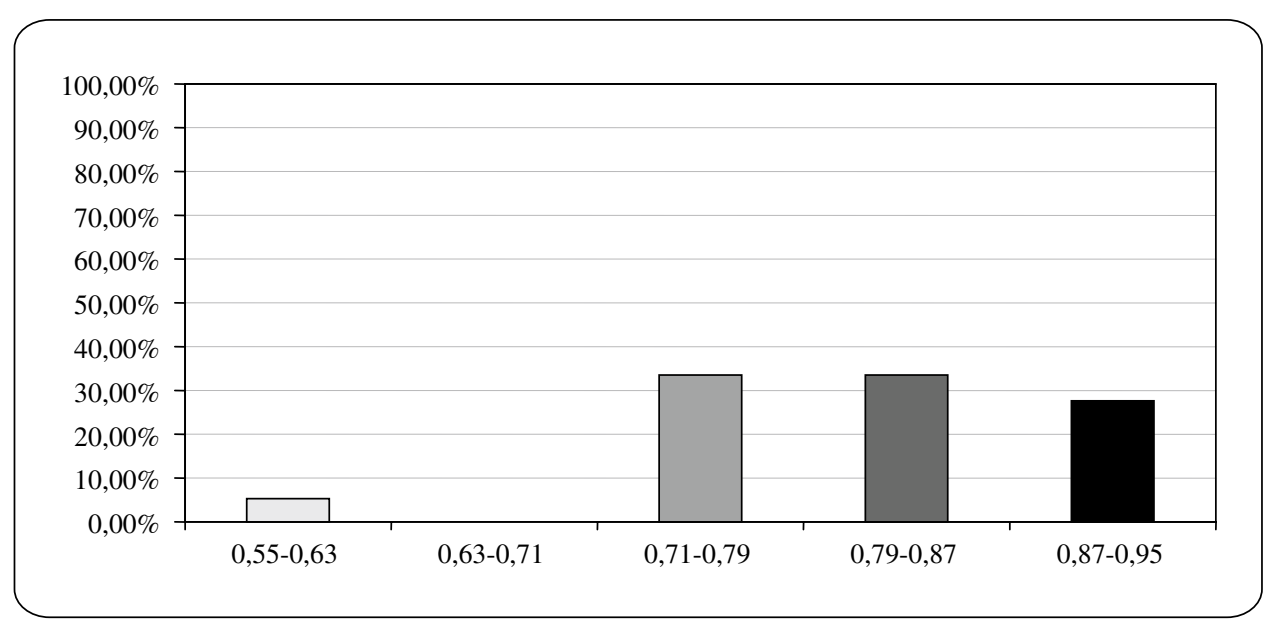

Cabe destacar que existen seis (6) educandos que tienen poca vulnerabilidad y requieren de un proceso educativo y pedagógico que se concentre o focalice en las dimensiones pedagógicas más afectadas o que los hacen más vulnerables. En cinco de estos casos $(83,3 \%)$, las variables que más los afectan son el sujeto que enseña o profesor y el contexto familiar. Llama la atención que en este grupo de párvulos con poca vulnerabilidad, 4 de 6 educandos $(66,6 \%)$ tienen más de dos dimensiones que les afectan.

El IVEA en Primer Ciclo de Educación Básica. En EBA se encuentra como valor mínimo del Indice un 50,7\%, mientras que el valor máximo encontrado es de 90,4\%. Para observar el comportamiento del Indice en el nivel y su distribución, se fijan in- 
tervalos, calculando la diferencia entre los dos valores indicados (máximo y mínimo), para luego dividirlo por 5, lo que entrega la amplitud del intervalo. Al igual que para el nivel anterior, mientras mayor es el porcentaje obtenido por el aprendiz menor es su vulnerabilidad educativa.

$$
\begin{gathered}
\text { Max }- \text { Min = Rango } \\
\text { Rango } / 5=\text { Amplitud }
\end{gathered}
$$

\begin{tabular}{|c|c|c|c|c|}
\hline Intervalo & Quintil & $\begin{array}{c}\text { Nivel de } \\
\text { Vulnerabilidad }\end{array}$ & Aprendices & Porcentaje \\
\hline $0,50-0,58$ & primero & $\begin{array}{c}\text { Totalmente } \\
\text { Vulnerables }\end{array}$ & 12 & $3,40 \%$ \\
\hline $0,58-0,66$ & segundo & $\begin{array}{c}\text { Altamente } \\
\text { Vulnerables }\end{array}$ & 19 & $5,39 \%$ \\
\hline $0,66-0,74$ & tercero & Vulnerables & 94 & $26,70 \%$ \\
\hline $0,74-0,82$ & cuarto & Poco Vulnerables & 177 & $50,28 \%$ \\
\hline $0,82-0,90$ & quinto & No Vulnerables & 50 & $14,20 \%$ \\
\hline & & & 352 & $99,97 \%$ \\
\hline
\end{tabular}

Distribución de aprendices de EBA según

Indice de Vulnerabilidad Educativa (IVE)

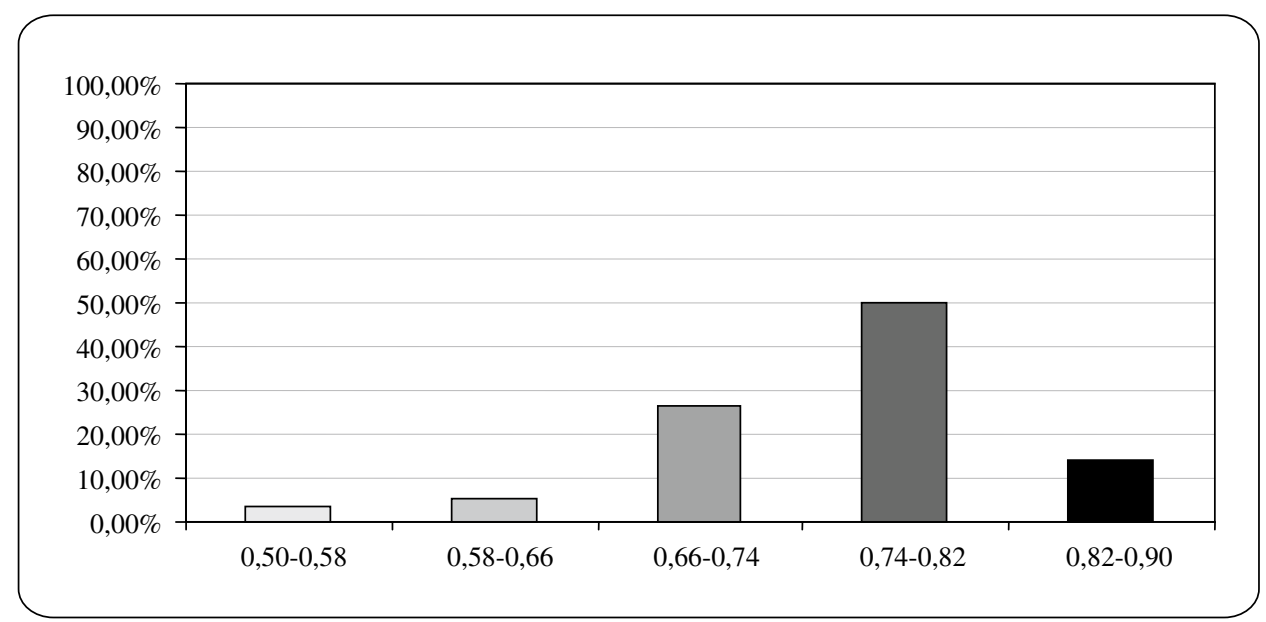

Es posible observar en el gráfico que 266 educandos de EBA, equivalentes a un $64,2 \%$, presentan un IVEA superior a un $66 \%$, concentrados desde el tercer intervalo, lo cual nos indica que tienen en su mayoría una baja vulnerabilidad educativa. Sin embargo, al igual que en el nivel de educación parvularia, también se advierte una diversidad de situaciones educativas. En efecto, 125 educandos, equivalentes a un $35,51 \%$ de la po- 
blación estudiada, son vulnerables en diferentes niveles, lo que puede ser observado en detalle en la base de datos construida para tales efectos y que no podemos hacer públicas por las razones indicadas.

Cabe señalar que los educandos que tienen poca vulnerabilidad y requieren de un proceso educativo y pedagógico que se concentre o focalice en las dimensiones pedagógicas más afectadas o que los hacen ser más vulnerables, son 176 de un total de 352 en este nivel, lo que equivale a un 50,0\% de la población estudiada. Las dimensiones que hacen más vulnerables a estos niños y niñas son las que dicen relación con el sujeto que enseña o el profesor, seguida por la de su contexto familiar. Con la primera, un $73,2 \%$ (129) de los educandos poco vulnerables se ven afectados y con la segunda un 59,0\% (104). Por otra parte, cabe destacar que en EBA 32 aprendices $(18,1 \%)$ de los 176 educandos con poca vulnerabilidad educativa, presentan al mismo tiempo tres dimensiones que les afectan en su vulnerabilidad educativa, lo que complejiza el proceso pedagógico para satisfacer sus diversas necesidades educativas.

\section{CONCLUSION}

A modo de conclusión se responderá la pregunta que se dejó pendiente:

¿Todos los niños y niñas de familias que pertenecen al Programa Puente de la comuna de Valparaíso, que cursan segundo ciclo de educación parvularia y primer ciclo de educación básica, tienen alta vulnerabilidad educativa?

Sobre la base de la información arrojada por el IVEA es posible responder que NO. No todos estos aprendices tienen una alta vulnerabilidad educativa, aunque sí tienen una alta vulnerabilidad socioeconómica. Por el contrario, se observa en ellos y ellas una diversidad de situaciones escolares y niveles de vulnerabilidad educativa, fenómeno que ocurre en ambos niveles educativos.

En los educandos de ambos niveles educativos se da una distribución normal, en la que la minoría se concentra en situaciones totalmente vulnerables, alta vulnerabilidad o de vulnerabilidad; la mayoría son poco vulnerables y otros son definitivamente no vulnerables. Sin embargo, tal como se presentó en los gráficos, se advierte que en el primer ciclo de educación básica habría mayor cantidad de educandos con vulnerabilidad educativa en sus diferentes niveles, lo que en términos de política es un desafío en tanto la formación en estos primeros años es fundamental para sustentar desarrollos escolares posteriores.

Dicho en positivo, los educandos estudiados presentan una diversidad de situaciones escolares que los hacen ser más o menos vulnerables aunque todos y todas provengan de familias de estratos socioeconómicos bajos y con una alta vulnerabilidad social. Vale decir, al igual que en otros estratos sociales, el nivel socioeconómico no es una variable que por sí sola incide directamente en los procesos educativos. Entonces, al igual que en otros estratos sociales, en los bajos existen educandos que tienen mayor o menor fragilidad respecto de fracasar en el sistema escolar con los requerimientos que éste exige, y en el caso de los 370 educandos estudiados, sólo la minoría tiene situaciones de total y alta vulnerabilidad. 
Dado lo expuesto y sobre la base de la evidencia recogida, es posible afirmar que la relación existente entre la vulnerabilidad socioeconómica y vulnerabilidad educativa, en estos aprendices, NO es una relación directa, ni unicausal y tampoco necesariamente negativa.

Otra conclusión posible de establecer a partir del estudio, aunque no da cuenta de la pregunta central, pero que parece necesario explicitar, es la incidencia de la variable información en el proceso y resultado de la investigación. Si bien la mayoría de los establecimientos con los que se trabajó poseen información de sus estudiantes, profesores y de su gestión pedagógica, la mayoría no cuenta con información organizada y tampoco integrada y mucho menos informatizada. En efecto, el acceso a dicha información y el manejo de la misma se hizo más o menos expedito según si las escuelas contaban con ella de manera organizada e integrada, ya sea en archivos manuales, informatizados y, en el mejor de los casos, en bases de datos. Cabe señalar que la minoría de las escuelas contó con un sistema organizado y asequible de información integrada. La información se maneja de manera parcelada a lo menos en dos ámbitos, información social e información pedagógica. Sin embargo, el educando es un ser integral, por lo que se sugiere implementar un sistema integrado e informatizado (biopsicosocial y pedagógico) de profesores y educandos, para mejorar la gestión pedagógica del establecimiento. Tal sugerencia se entrega, en tanto posibilita re-conocer la identidad de todos y cada uno de los niños y niñas bajo su responsabilidad; favorece procesos de evaluación diagnóstica integral y permite re-conocer la diversidad de necesidades educativas que se configuran en ellos y ellas; y porque, en definitiva, el manejo de este tipo de información favorece la toma de decisiones en el proceso pedagógico de cada educando, de cada aula y de cada escuela. En este mismo sentido, y por las mismas razones esgrimidas, se sugiere a los organismos públicos integrar y digitalizar (a quienes no lo tengan) la información infantil que actualmente se evidenció compartimentalizada por sectores y niveles.

Finalmente cabe destacar la gran importancia que revisten en la contención de los problemas sociales de los niños y niñas el Programa Puente y los programas de auxilio escolar de la JUNAEB. Se advierte, por la información recogida, que ambas instituciones cumplen un rol significativo en paliar la precariedad de las variables sociales en juego. Sin embargo, queda pendiente que estas variables sean integradas en el proceso pedagógico que debe asumir el sector educación, tanto a nivel de política pública, en el nivel de educación parvularia y especialmente en el de educación básica, como en la contextualización y materialización de dicha política, en el proceso pedagógico de cada escuela y, más específicamente, de cada aula, en los que los profesionales de la educación tienen la máxima responsabilidad.

\section{BIBLIOGRAFIA}

Álvarez, Silvana y otras (2006). La Función del profesor de Educación Diferencial en la evaluación de niños, niñas y adolescentes en vulnerabilidad social en instancias de educación no formal: Un estudio cualitativo. Trabajo de Titulación para optar al título de profesor de Educación Diferencial y al grado de Licenciado en Educación. Pontificia Universidad Católica de Valparaíso. Profesora Guía, Cristina Julio Maturana.

Alegre de la Rosa, Olga María (2000). Diversidad Humana y Educación. Málaga, España: Ediciones Aljibe. 
Bachelet, Michelle (2006). Discurso presidencial del 21 de mayo de S.E. la Presidenta de la República. http://www.gobiernodechile.cl/21 mayo2006/indice_discursos.asp

Bellei, Cristián y otros (2003). ¿Quién dijo que no se puede? Escuelas efectivas en sectores de pobreza. Santiago, Chile: UNICEF.

Comisión de Expertos (2004). Nueva perspectiva y Visión de la Educación Especial. Informe. Santiago de Chile: Mineduc.

Da Costa, Maria (2005). Conferencia: Los desafíos de la formación docente para una educación infantil del siglo XXI: post estructuralismo, globalización y sus implicaciones para la educación de la primera infancia. En Seminario Internacional: Construyendo el futuro de los niños y niñas desarrollado en noviembre.

Dávila; Ghiardo y Medrano (2005). Los Desheredados. Trayectorias de vida y nuevas condiciones juveniles. Viña del Mar: CIDPA ediciones.

Devalle, Alicia y Viviana Vega (1999). Una escuela en y para la diversidad. El entramado de la diversidad. Argentina: Aique.

González, Roxana y otras (2005). Descripción del sistema de redes entre Escuelas Básicas y de Lenguaje en el marco de los procesos de Integración de niños y niñas con trastornos específicos del lenguaje en edad posterior a los 5 años, 11 meses: Un estudio exploratorio. Trabajo de titulación para optar al título de profesor de Educación Diferencial y al grado de Licenciado en Educación. Pontificia Universidad Católica de Valparaíso. Profesora Guía, Cristina Julio Maturana.

Julio, Cristina (2004a). Informe del Proyecto DEI-PUCV N $188.734 / 2003$ titulado Período de 'marcha blanca': estudio de caso de una escuela de lenguaje que inicia un proceso de implementación gradual de los nuevos planes y programas para alumnos y alumnas con trastornos específicos del lenguaje (Decreto $\left.\mathrm{N}^{\circ} 1300 / 2002\right)$.

Julio, Cristina (2004b). ¿Escuela Especial de Lenguaje o Jardín Infantil Regular?: Una tensión para la enseñanza de párvulos con formas peculiares de lenguaje. Ponencia presentada en el Congreso Internacional de Atención a la Diversidad en Educación Parvularia. Universidad de la Frontera. Temuco.

Julio, Cristina (2005). Niños, niñas y adolescentes fuera del sistema escolar: diversidad injusta y un compromiso pendiente en la política de educación especial. Ponencia presentada en el IV Encuentro Internacional de Educación Especial y Psicopedagogía. Universidad Católica del Maule. Talca.

Julio, Cristina (2006). Informe Final de Asesoría Técnico Pedagógica a proyectos de reescolarización comunitaria del Centro Juvenil El Puerto y la Corporación Servicio Paz y Justicia (SERPAJ), en el contexto del Convenio Ministerio de Educación y la Pontificia Universidad Católica de Valparaíso.

Lecannelier, Felipe (2006). Apego e Intersubjetividad. Influencia de los vínculos tempranos en el desarrollo humano y la salud mental. Santiago, Chile: LOM editores. Serie Universitaria.

Ley Orgánica Constitucional de Enseñanza (LOCE 1990).

López, Gabriela; Jenny Assael y Elisa Neumann (1991). La Cultura Escolar ¿Responsable del Fracaso? Estudio Etnográfico en dos escuelas urbano populares. Santiago: Programa Interdisciplinario de Investigación en Educación (PIIE).

Márquez, Rodrigo (2006). El Diseño de índices sintéticos a partir de datos secundarios: Metodologías y Estrategias para el análisis social. En Canales, Manuel (coordinador y editor), Metodologías de Investigación Social. Santiago: LOM.

Ministerio de Educación (2005a). Plan de Apoyo a iniciativas de reescolarización orientadas a la población con alta vulnerabilidad social. Programa Intersectorial de Reescolarización. Documento de trabajo Mesa Técnica de Universidades.

Ministerio de Educación (2005b). Guías de apoyo a la escuela. Material de apoyo sobre la investigación UNICEF 2003 ¿Quién dijo que no se puede? 
Ministerio de Educación (2005c). Nuestro Compromiso con la Diversidad. Política Nacional de Educación Especial. Santiago: Serie Bicentenario.

Ministerio de Educación (2006). Seminario Educación de Calidad para un Buen Comienzo.

Ministerio de Educación-CPEIP (2006). Construcción de saberes pedagógicos desde la Implementación de la Reforma en Educación Parvularia.

Núñez, Iván (1995). La Educación Chilena en el período 1945-1990. En Puiggrós, Adriana y Lozano, Claudio (Coord.). Historia de la Educación Iberoamericana: Argentina, Bolivia, Brasil, Caribe, Centroamérica, Cuba, Chile, España. (pp. 231-252).

Peralta, María Victoria (2002). Una Pedagogía de las oportunidades. Nuevas Ventanas para los Párvulos Latinoamericanos del siglo XXI. Santiago de Chile: Editorial Andrés Bello.

Rogoff, Bárbara (1993). Aprendices del Pensamiento. El desarrollo cognitivo en el contexto social. Barcelona: Paidós.

Rosenthal, Robert (1980). Pigmalión en la Escuela: Expectativas del Maestro y Desarrollo Intelectual del Alumno. Madrid, España: Marova.

Tezanos, Araceli (1992). Acerca de los Significados de Educación y Pedagogía. Aportes para una reforma al sistema educativo. Santiago de Chile. Apuntes de la autora.

UNESCO (1990). Declaración Mundial sobre Educación Para Todos. La satisfacción de las Necesidades Básicas de Aprendizaje. Conferencia Mundial de Jomtien.

UNESCO (1994). Declaración de Salamanca y Marco de Acción Sobre Necesidades Educativas Especiales. Conferencia Mundial sobre Necesidades Educativas Especiales: Acceso y Calidad. Salamanca, España.

UNESCO (2000). Índice de Inclusión.

UNESCO/OREALC (2004). Temario Abierto sobre Educación Inclusiva. Materiales de Apoyo para Responsables de Políticas Educativas.

Unidad de Currículum y Evaluación UCE (2000 y 2002). Escuelas Testigo. Documento de Trabajo interno del Ministerio de Educación. 